\title{
A forma do arco dentário inferior na visão da literatura
}

\author{
Tarcila Triviño*, Danilo Furquim Siqueira**, Marco Antonio Scanavini***
}

\begin{abstract}
Resumo
Objetivo: esse trabalho teve como objetivo principal analisar a literatura relacionada a um relevante aspecto clínico, a configuração do arco dentário, salientando a importância da manutenção da forma original do arco dentário para se obter a estabilidade do tratamento ortodôntico, bem como avaliar os métodos utilizados para a determinação da forma do arco e os diagramas de forma propostos por diversos autores. Resultados e Conclusões: constatou-se uma controvérsia quanto à indicação de uma forma ideal média e a seleção de uma forma individualizada a partir de diagramas que forneçam configurações variadas para o arco dentário. Entretanto, observou-se um progresso nas metodologias aplicadas nos recentes estudos, o que possivelmente fornecerá precisão nos próximos resultados.
\end{abstract}

Palavras-chave: Arco dentário. Forma do arco. Estabilidade. Ortodontia.

\section{INTRODUÇÃO}

A prática clínica da Ortodontia tem como fundamentos principais a busca pelo equilibrio entre as estruturas craniofaciais e pela estabilidade da oclusão, aspectos esses que estão correlacionados a fatores como as características anatômicas e o comportamento do arco dentário inferior, os quais devem ser analisados com critério quando se tem por finalidade alcançar estética e função adequadas.

Em um tratamento ortodôntico, as posições dentárias serão estabelecidas, basicamente, pela configuração do arco ósseo basal, ou seja, os den- tes serão alinhados sob o rebordo alveolar, o qual apresenta uma forma específica.

O arco ósseo basal é estabilizado em mais ou menos 9,5 semanas de vida intra-uterina ${ }^{9}$. Sua forma é, inicialmente, determinada pela configuração do suporte ósseo e, em seguida, pela erupção dos dentes, pela musculatura peribucal e pelas forças funcionais intrabucais ${ }^{8}$. Devido à influência de vários fatores, a estabilidade das posições dentárias é um dos grandes desafios enfrentados pelos profissionais ao realizarem uma intervenção ortodôntica.

* Especialista em Ortodontia pela Universidade Federal Fluminense (UFF). Mestre em Odontologia, área de concentração Ortodontia, pela Universidade Metodista de São Paulo (UMESP).

** Mestre e Doutor em Ortodontia pela FOB-USP. Professor do Programa de pós-graduação em Odontologia, área de concentração Ortodontia, da Universidade Metodista de São Paulo (UMESP).

*** Coordenador e Professor do Programa de pós-graduação em Odontologia, área de concentração Ortodontia, da Universidade Metodista de São Paulo (UMESP) 
A chave para o sucesso ou insucesso da conservação das novas posições dentárias estabelecidas com a terapia parece estar relacionada com o posicionamento correto dos dentes nas bases apicais, com a preservação das dimensões transversais do arco dentário ${ }^{28,52}$ e, sobretudo, com a manutenção do equilíbrio funcional dos músculos da face.

Sendo assim, com o intuito de evitar a recidiva, estudos aconselham a considerar, no planejamento e no tratamento ortodôntico, aspectos como a não alteração da forma original do arco dentário por meio da expansão ou contração não apenas das distâncias intercaninos e intermolares ${ }^{31,43,52,53}$, mas também da largura interpré-molares ${ }^{12,24,39}$, principalmente dos dentes inferiores, o que tem conduzido os autores a referenciarem o arco dentário inferior como um dos principais elementos para o diagnóstico e a terapia ortodôntica ${ }^{11,40,42} \mathrm{e}$ a realizarem pesquisas com o objetivo de definir o tamanho e formas ideais para essa estrutura.

A arcada humana apresenta extensas variações individuais quanto à sua configuração e, por sua vez, essa vem sendo descrita por formas geométricas, fórmulas matemáticas e métodos computadorizados desenvolvidos com a finalidade de facilitar ou tornar mais didática a representação da forma do arco dentário.

Baseado na importância da forma e dimensões originais do arco dentário inferior no tratamento ortodôntico e na estabilidade dos resultados por ele atingidos, esse trabalho objetiva revisar a literatura, abordando aspectos da relevância quanto à manutenção da dimensão transversal e a forma do arco, bem como, apresentar as formas geométricas e métodos empregados pelos pesquisadores na determinação e descrição da configuração do arco dentário.

\section{REVISÃO DE LITERATURA}

Nos meados do século XVIII, surgia, tologia, uma especialidade que tinha como objetivo principal a correção das posições dos dentes que apresentavam-se em uma posição inadequada quanto ao seu alinhamento no osso alveolar. Devido a esse aspecto, essa ciência foi denominada Ortodontia, originada das palavras gregas orthós (reto) e odóntos (dentes).

No início dessa prática odontológica, os profissionais realizavam a correção das más posições dentárias com movimentos aleatórios dos dentes para vestibular e ou para a lingual, feitos sem nenhuma restrição, ou seja, sob os ossos alveolares, os dentes eram recolocados em novas posições, de modo que esses ficassem alinhados no rebordo alveolar. $\mathrm{Na}$ maioria dos tratamentos, para se atingir o objetivo de um adequado alinhamento dentário, preconizava-se a expansão ou contração do arco dentário.

Contudo, com o decorrer do tempo, os profissionais passaram a observar que os resultados alcançados com a terapia ortodôntica, baseada nessa filosofia, não se mostravam estáveis no período pós-tratamento, ou seja, as posições irregulares dos dentes reincidiam ${ }^{55}$.

Esse aspecto despertou, entre os especialistas ${ }^{31,}$ $43,52,53,55$, o interesse em estudar as possíveis causas da recidiva dos seus tratamentos. Notou-se que as alterações transversais provocadas no arco dentário eram temporariamente mantidas, somente enquanto os aparelhos de contenção estavam sendo utilizados. Após sua remoção, na maioria dos seus pacientes, os dentes tendiam a assumir suas posições originais ${ }^{23,28,41,55}$, ou seja, as distâncias entre os dentes homólogos retornavam aos valores do início do tratamento, reincidindo o apinhamento ou o espaçamento dentário decorrentes das discrepâncias entre as bases ósseas e os dentes. Ainda, quanto maiores fossem as alterações feitas na forma do arco dentário durante a terapia empregada, maior seria a tendência ao colapso pós-tratamento ${ }^{47,50,64}$.

Segundo Brader ${ }^{7}$; Guerra ${ }^{19}$; Lear, Moorrees ${ }^{30}$ e Strang ${ }^{52,53}$, isso ocorria devido à não preservação do sistema balanceado de forças entre as estruturas dentárias, musculares - principalmente bochechas, lábios e língua - e ósseas, existente tanto na má oclusão como na oclusão normal, sendo inerente ao indivíduo. 
Com base nas observações obtidas por esses autores ${ }^{7,19,30,52,53}$, pode-se mencionar que as alterações provocadas pela mecânica ortodôntica devem ser feitas de modo a não afetar o equilibrio entre as estruturas constituintes do sistema estomatognático, pois a preservação desse equilibrio será um dos fatores de maior importância no sucesso do tratamento, ou seja, na estabilidade dos resultados atingidos com a terapia aplicada.

Essa teoria foi comprovada por outros autores $^{23,28,48,50,52}$, que observaram a possível manutenção de alguma quantidade de expansão ou de contração do arco dentário após o tratamento, apenas quando as posições dentárias não alterassem a função fisiológica dos músculos e, de acordo com demais autores ${ }^{1,11,23,53,54}$, nos casos em que houvesse a necessidade de se fazer alguma movimentação no sentido vestibulolingual para se obter um correto alinhamento dos dentes no arco e um adequado relacionamento com os dentes adjacentes. $\mathrm{O}$ limite desse reposicionamento lateral é orientado e estabelecido pelo osso alveolar dessa região (borda WALA) ${ }^{1,11}$.

Pode-se, então, afirmar que o movimento ortodôntico será limitado pelas estruturas ósseas, musculares e pelos tecidos moles adjacentes ${ }^{60}$ e que os dentes não são estabilizados na nova posição se não houver equilíbrio entre essas estruturas ${ }^{50}$. Conseqüentemente, as forças musculares são os fatores determinantes das posições dentárias no osso alveolar e estabelecerão uma forma individual para o arco dentário $28,30,52,53,60$.

Em busca de excelência em seus resultados, os ortodontistas começaram a considerar os conceitos de equilíbrio e a planejar o tratamento ortodôntico respeitando o limite do arco inferior ${ }^{1,11,42,52}$. Passaram a relevar a necessidade de se manter a forma do arco dentário do início ao final do tratamento, ou melhor, dar importância à configuração inicial do arco dentário ao longo de toda a terapia ortodôntica, pois se certificaram de que esse fator tinha grande influência nos resultados e na estabilidade de seus casos tratados $2,5,20,34,41,54$.
Sendo assim, uma vez que já compreendiam que a terapia ortodôntica deveria respeitar e preservar os limites das medidas da largura do arco dentário, os profissionais passaram a realizar estudos com o objetivo de elaborar uma representação do arco dentário que possibilitasse acesso imediato, durabilidade e precisão, e com isso estabelecer um padrão de forma, o qual seria utilizado como base para o reposicionamento adequado dos dentes.

Alguns autores ${ }^{1,18,54}$ aconselharam a utilização de protótipos ou referências que auxiliassem o profissional durante todo o tratamento ortodôntico, especificamente para a confecção dos arcos metálicos e quanto à seleção dos arcos metálicos pré-formados. Esses parâmetros seriam obtidos por meio da utilização dos modelos iniciais do paciente ${ }^{1,54}$, ao longo do tratamento ortodôntico, os quais forneceriam a forma do rebordo alveolar ${ }^{1,18,54}$, utilizada na confecção dos arcos metálicos. Desse modo, evitarse-ia as alterações transversais no arco dentário, referenciando as medidas das distâncias transversais do arco. Todavia, a manipulação, na prática clínica, dos modelos de gesso poderia não ser prática, onde muitas vezes ocorriam fraturas desses elementos de diagnóstico ${ }^{26}$.

Concomitantemente, observa-se uma constante preocupação por parte dos autores que realizaram estudos com o objetivo principal de desenvolver métodos que descrevessem a representação de uma forma ideal para o arco dentário. Inicialmente, os padrões idealizados nos estudos consistiam, basicamente, na descrição da forma por meio da utilização de figuras geométricas, as quais forneceriam uma representação adequada da configuração do arco dentário.

Alguns autores associaram, simplesmente, a forma do arco dentário a figuras geométricas, como a parábola $2,4,14,51$, a curva catenária ${ }^{9,16,29,34,36,38,46}$, a elipse $e^{4,14,27,36}$, a forma em "U"39 ou um segmento de círculo unido a linhas retas ${ }^{6,22}$ ou ainda esferas modificadas ${ }^{35}$.

Entretanto, com a associação de duas ou mais figuras geométricas, foram desenvolvidos desenhos 
construídos com o emprego de traçados geométricos realizados com base em medidas descritivas do crânio, da face e dos elementos que compõem os arcos dentários ${ }^{35}$, os quais foram denominados diagramas. Esses, por sua vez, foram considerados métodos que facilitariam ou tornariam mais didática a representação da forma do arco e atuariam como guia ${ }^{20}$ durante o tratamento ortodôntico. Assim, com a utilização de um diagrama personalizado para o paciente, os arcos metálicos seriam confeccionados com formato e dimensões padronizados e constantes, permitindo a manutenção das distâncias transversais e ântero-posteriores durante o tratamento.

A utilização das dimensões da face e do crânio na descrição de seus diagramas de forma do arco foi preconizada por alguns autores ${ }^{6,7,27}$ pois, segundo eles, a forma do arco variaria conforme o padrão facial e o tipo craniano ${ }^{67}$.

Um dos percussores na idealização de diagramas de forma de arco foi o protesista Bonwill ${ }^{6}$, que em 1885, com a utilização da distância intercondilar de 4 polegadas e a soma das medidas dos 6 dentes anteriores, construiu um desenho

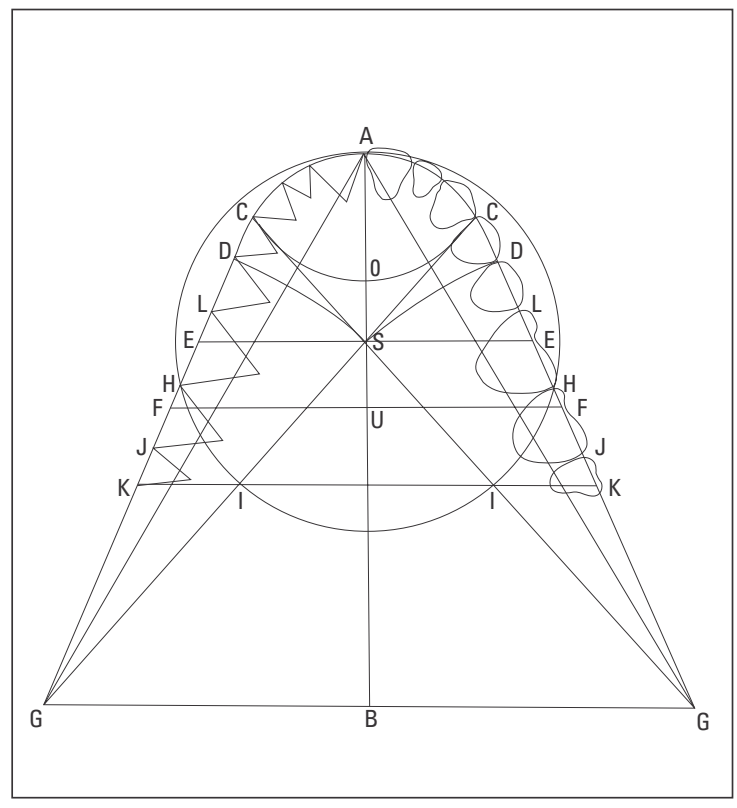

FIGURA 1 - Diagrama de Bonwill'. geométrico baseado em uma circunferência, cujo raio equivaleria à soma dos 6 dentes anteriores, e em um triângulo eqüilátero, cuja base seria a distância intercondilar do paciente (Fig. 1). Segundo o diagrama de Bonwill ${ }^{6}$, os incisivos estariam posicionados em um semicírculo, enquanto os dentes posteriores em uma linha reta.

Todavia, mesmo com o emprego de medidas semelhantes, como as dimensões das estruturas faciais, a forma do arco não foi descrita igualmente entre os autores. Em 1927, Izard ${ }^{27}$ acreditava ter uma razão constante entre a largura do arco e a largura da face, bem como o comprimento do arco e o comprimento da face. Utilizou o arco superior na determinação do arco ideal e considerou que as variações em forma do arco dentário poderiam ser descritas por uma elipse (Fig. 2).

Posteriormente, Brader ${ }^{7}$ (1972) afirmou que a forma do arco dentário seria a representação de um equilíbrio entre as forças da língua e a musculatura peribucal, estando associada à uma elipse. Descreveu um diagrama baseado na equação $P R=C$, onde $\mathrm{P}$ é igual à pressão por unidade de área, $\mathrm{R}$ é o raio da curvatura elíptica e $C$ é uma constante ma-

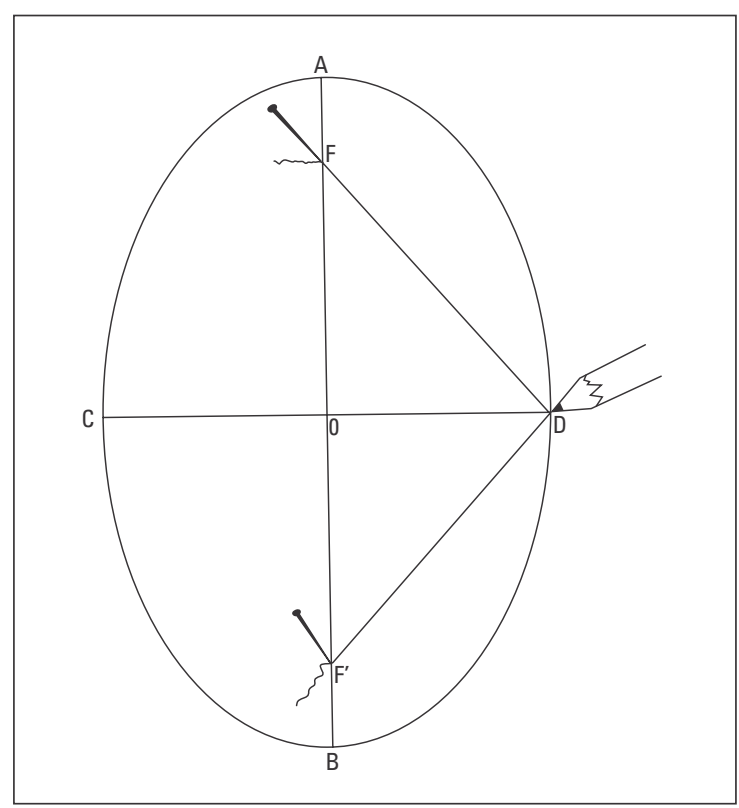

FIGURA 2 - Diagrama de Izard²7. 
temática e, a partir desse, estabeleceu formas com distâncias intermolares de $48 \mathrm{~mm}$ a $68 \mathrm{~mm}$ (Fig. 3).

Embora tenham sido utilizadas medidas faciais semelhantes, a ocorrência de diversidade entre os autores acima mencionados ${ }^{6,7,27}$ quanto à configuração ideal do arco dentário, pode ser devida ao emprego de medidas base, as quais deveriam ser feitas com o máximo de atenção, cuidado e precisão, pois o mínimo erro na sua obtenção resultaria num desenho distinto e/ou inadequado.

Essa dificuldade levou, então, à idealização de diagramas ou formas baseadas apenas em medidas obtidas a partir dos dentes, uma vez que seriam esses os recolocados sob o rebordo ósseo com formato pré-estabelecido na fase embrionária ${ }^{9}$. Sen-

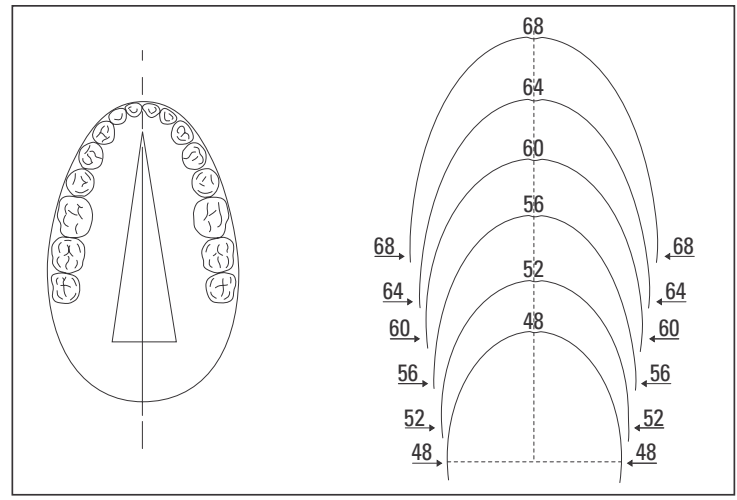

FIGURA 3 - Diagrama e formas de Brader?

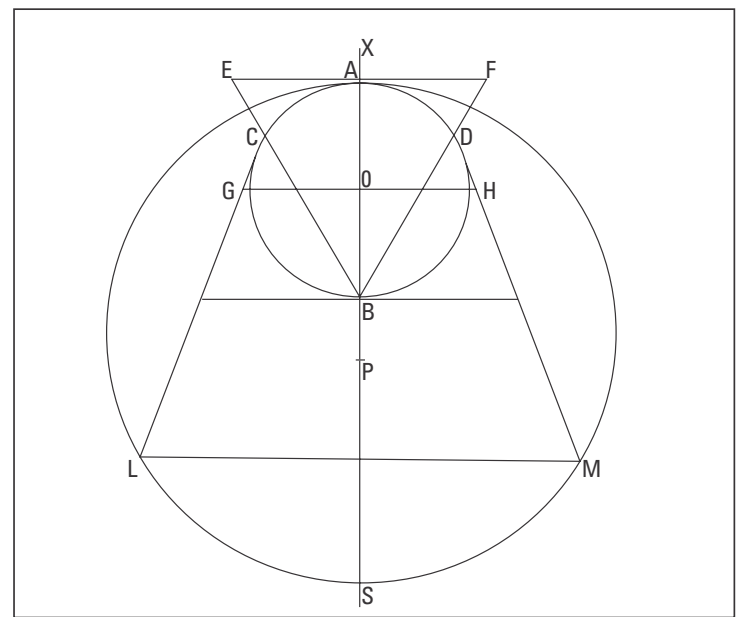

FIGURA 4 - Diagrama de Hawley ${ }^{20}$. do assim, Hawley ${ }^{20}$ (1905) idealizou um diagrama muito semelhante ao de Bonwill ${ }^{6}$, construído apenas com a medida da soma do diâmetro mesiodistal dos 6 dentes anteriores inferiores (Fig. 4).

O diagrama de Bonwill-Hawley ${ }^{6,20}$ foi utilizado por muitos anos na prática da Ortodontia, sendo modificado ou aperfeiçoado por $\mathrm{Chuck}^{13}$ (1934) e Boone $^{5}$ (1963), que apenas o construíram em um papel milimetrado para facilitar a coordenação dos arcos (Fig. 5). Devido à sua relevância, esse diagrama foi ainda a base para o desenvolvimento de demais diagramas idealizados por Sved ${ }^{56}$ (1917), Gysi e Carrea, como descrito por Monti ${ }^{35}$ em 1958 e Interlandi ${ }^{26}$ (1978), representados nas figuras 6 a 9 .

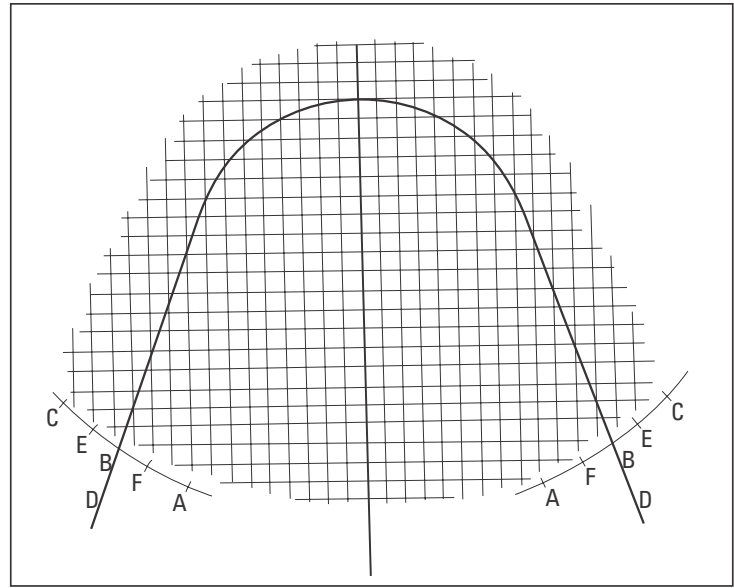

FIGURA 5 - Diagrama proposto por Boone 5 .

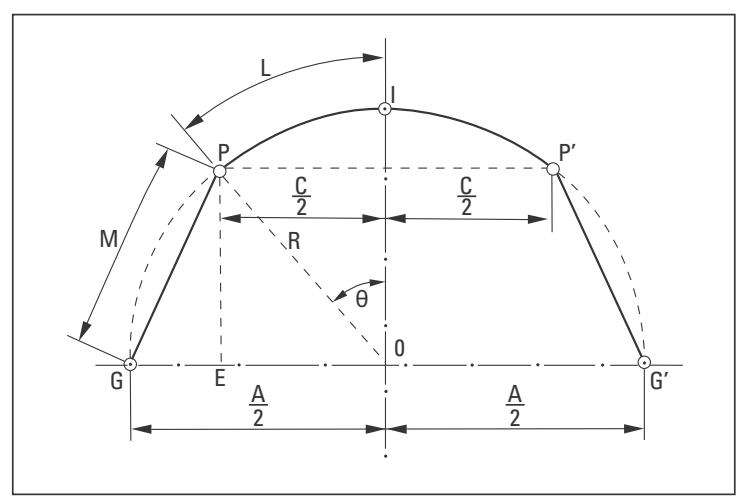

FIGURA 6 - Diagrama de Sved ${ }^{56}$. 
Contudo, em 1907, Angle², não concordando com a utilização do diagrama de Bonwill-Hawley ${ }^{6,20}$ como a forma do arco ideal, preconizou como base para a forma do arco dentário a Linha de Oclusão, a qual seria descrita pelo alinhamento harmonioso e balanceado dos dentes sob os rebordos ósseos e as estruturas adjacentes. Segundo Angle, essa linha seria semelhante a uma parábola, sendo esta forma, posteriormente, encontrada também no diagrama de Herbst, descrito por Monti ${ }^{35}$ (1958), Currier ${ }^{14}$ (1969) apenas para a maxila e por Figún e Garino ${ }^{18}$ (1994) para a mandíbula.

A essa linha foram incorporadas dobras de $1^{\text {a }}$ ordem, ou seja, off-set nos caninos, primeiro e segundo molares superiores e inferiores ${ }^{2,5,13,26,52}$, que promoveram uma melhor intercuspidação dos dentes superiores com os inferiores, alterando sua forma original (Fig. 10).

Entre as outras figuras geométricas, a forma elíptica não foi apenas observada por $\operatorname{Izard}^{27}$ (1927) e Brader ${ }^{7}$ (1972) como a representação da configuração do arco dentário, mas pelos diagramas de Weiss e Gaillard (apud MONTI ${ }^{35}$,1958), Currier $^{14}$ (1969), Vigorito ${ }^{62}$ (1986) e Cruz et al. ${ }^{15}$ (1995), que encontraram essa forma em 96\% da amostra por eles estudada.

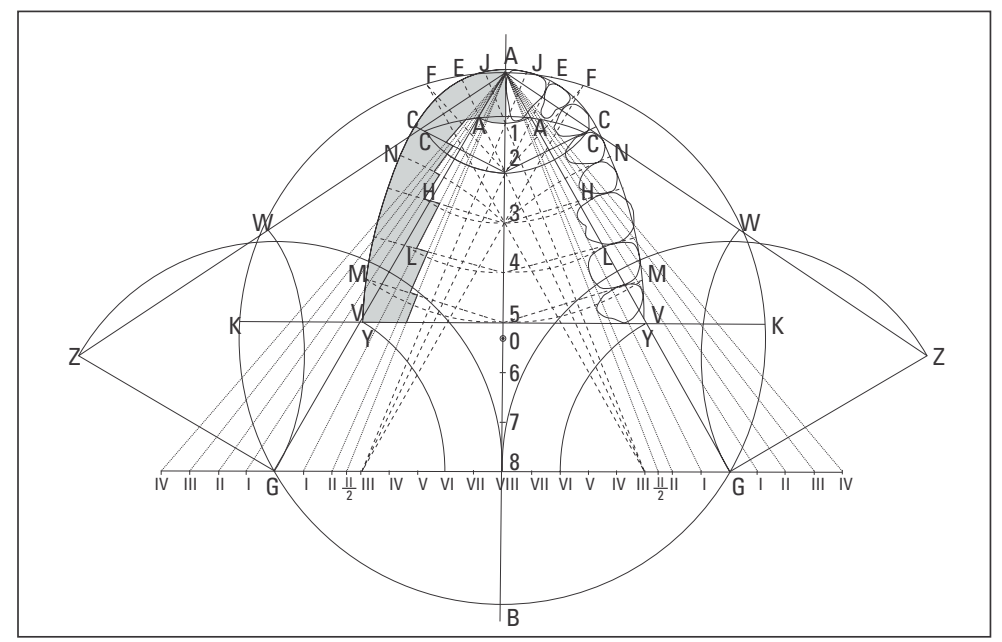

FIGURA 7 - Diagrama de Gysi ${ }^{35}$.

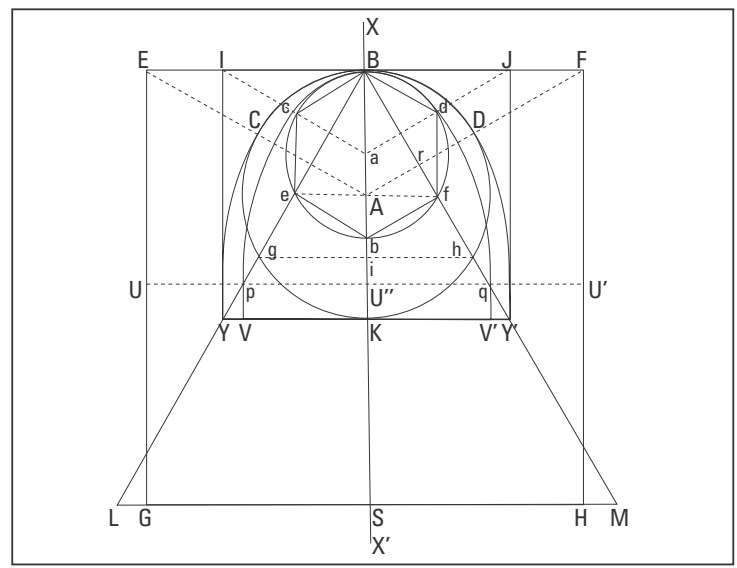

FIGURA 8 - Diagrama de Carrea ${ }^{35}$.

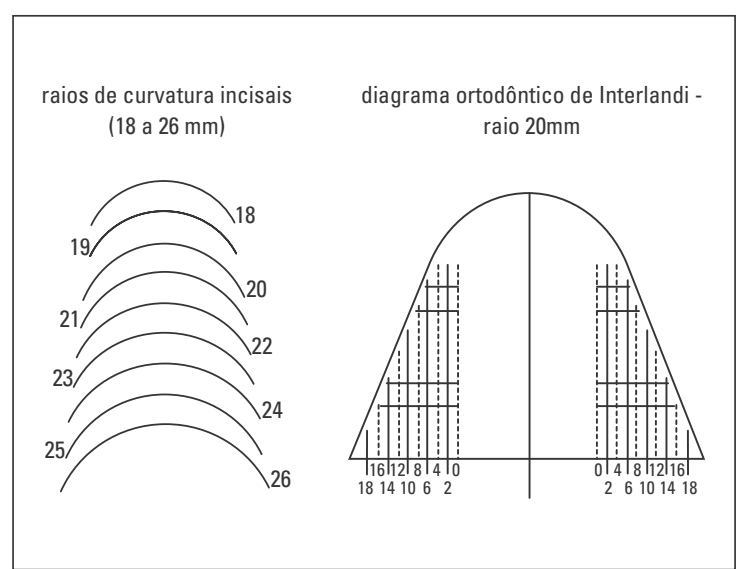

FIGURA 9 - Diagrama ortodôntico de Interlandi26. 
No entanto, Vigorito ${ }^{62}$ (1986) constatou que a maioria dos indivíduos da sua amostra não apresentava a forma de elipse, mas sim a forma catenária, a qual foi inicialmente descrita por $\operatorname{Scott}^{46}$ (1957), Burdi ${ }^{9}$ (1968) e Engel ${ }^{16}$ (1979), estabelecendo assim mais de uma representação de forma para o arco dentário.

$\mathrm{Na}$ literatura, observa-se que esse autor ${ }^{62}$ não foi o único a encontrar mais de uma forma para o arco dentário. De acordo com os resultados obtidos em vários estudos $4,10,18,25,34,37,39,40,42,45,49,57,58,59,65$, a curva do arco dentário não seria descrita, representada ou definida por apenas uma forma geométrica, mas sim por variadas formas, o que levou esses autores a desaconselharem a utilização de diagramas idealizados por outros pesquisadores $6,7,20,26,27,35,56$.

De acordo com os resultados de Weinberger ${ }^{65}$ (1914), os arcos de indivíduos com oclusão normal mostravam a região anterior em semicírculo e os dentes posteriores em linha reta, enquanto os arcos com má oclusão eram representados por 4 formas distintas, não sendo coincidentes com os conceitos de Ricketts ${ }^{42}$ (1979), que determinou 5 curvas distintas representativas do arco dentário inferior normal, as quais receberam denominações específicas, não associadas aos nomes de figuras

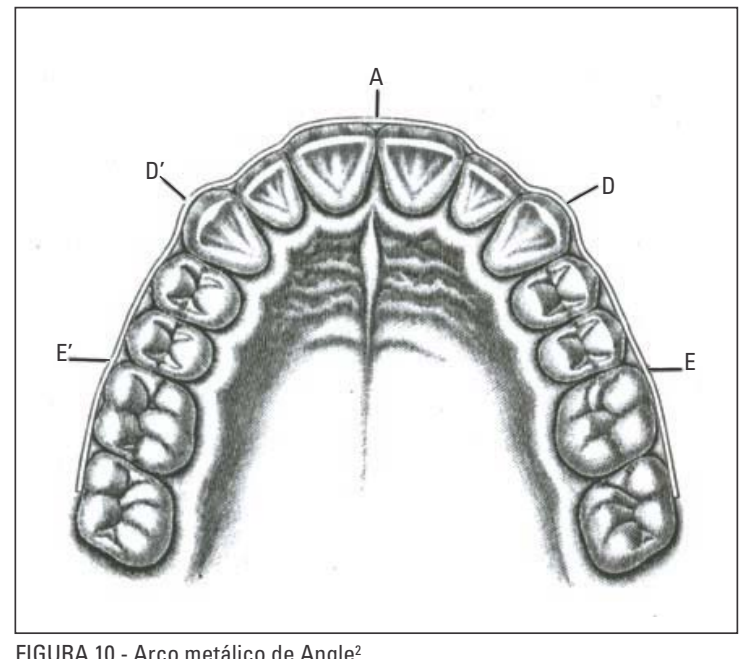

FIGURA 10 - Arco metálico de Angle². geométricas: normal, ovoid, tapered, narrow ovoid e narrow tapered (Fig. 11), sistema de classificação também empregado por outros autores ${ }^{40,57,58,59}$ para designar as formas por eles encontradas.

Posteriormente, Raberin et al. ${ }^{40}$, em 1993, também afirmou que a forma ideal do arco dentário com oclusão normal não seria uma forma simples e universal, mas no mínimo 5 formas diferentes, as quais denominou: narrow, wide, mid, pointed e flat (Fig. 12). No entanto, após a avaliação de modelos com oclusão normal de indivíduos brasileiros, Telles $^{57}$ (1991) observou 3 formas superiores e 3 formas inferiores, nomeadas triangular, oval e quadrada, com uma variação de tamanho, as quais constituíram um diagrama multiformétrico desenvolvido por ele (Fig. 13).

Recentemente, Capelozza Filho e Capelozza ${ }^{11}$ (2004) sugeriram o uso de um diagrama, denominado DIAO (diagrama individual anatômico objetivo), para a construção dos arcos metálicos a partir dos quais seria selecionada a forma do arco, de acordo com as características anatômicas de normalidade, com o diagnóstico e as metas do tratamento. A curvatura anterior do arco deveria incorporar os objetivos do tratamento quanto ao posicionamento dos incisivos e caninos, e a largura do arco expressar a movimentação desejável dos dentes (Fig. 14).

Apesar da concordância entre os autores quanto à diversidade nas formas que melhor descrevam o arco dentário, constataram-se pequenas diferenças encontradas por eles quanto ao número e os tipos de configurações para o arco dentário, o que pode ter sido induzido pelas distintas metodologias empregadas por esses autores ${ }^{11,40,42,57,58}$.

Sendo assim, tornou-se interessante avaliar mais detalhadamente a metodologia aplicada, nesses trabalhos, para a determinação da forma do arco dentário e, com isso, pôde-se notar uma certa preocupação por parte dos autores em empregar, cada vez mais, métodos precisos, capazes de fornecer a representação da forma do arco dentário com mais exatidão. 


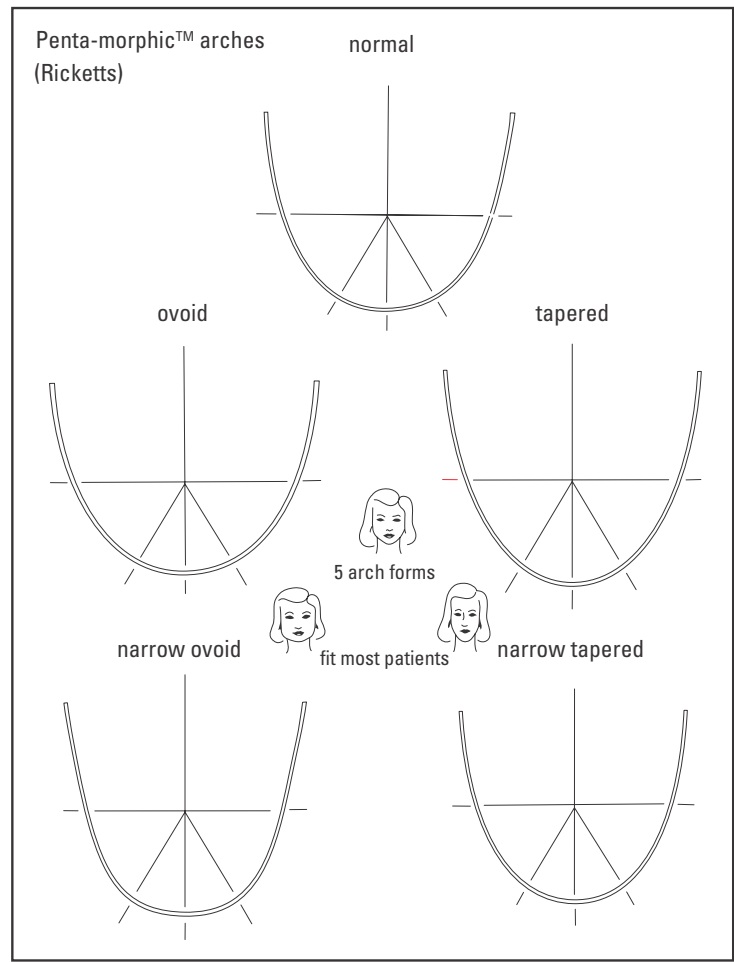

FIGURA 11 - Arcos pentamórficos de Ricketts $^{42}$.

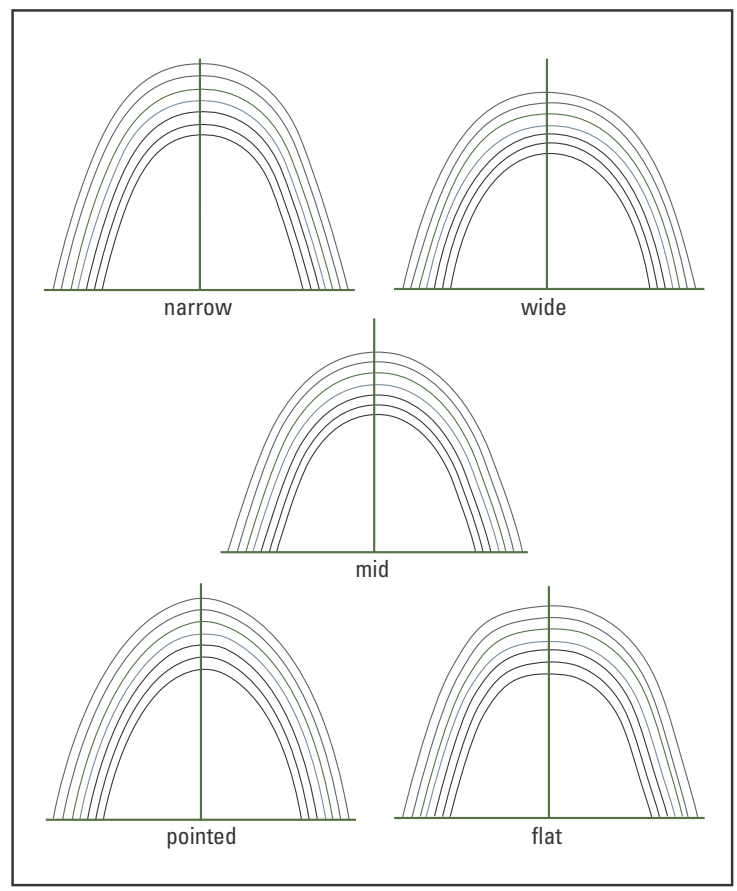

FIGURA 12 - Formas de Raberin ${ }^{40}$
De acordo com Staton ${ }^{49}$ (1922), qualquer método de determinação da forma do arco dentário deveria permitir a produção de formas como uma elipse, parábola, parábolas cúbicas, ferraduras e outras. Enfatizando esse conceito, em 1978, White $^{66}$ considerou a necessidade de uma fórmula matemática para determinar a forma do arco, devendo todo arco ideal ser simétrico e aderir a uma mesma forma, porém com tamanhos diferentes.

Essas considerações despertaram entre os autores o propósito de integrar ao método de determinação da forma do arco dentário o emprego de uma função algébrica ou geométrica, o que poderia tornar a representação da configuração do arco dentário mais fiel, uma vez que seria descrita por uma ciência exata, a matemática.

Com essa finalidade, a equação quadrática desenvolvida por Biggerstaff ${ }^{4}(1972) \mathrm{Ax}^{2}+\mathrm{By}^{2}+\mathrm{Cxy}$ $+\mathrm{Dy}+\mathrm{Ey}-\mathrm{F}=0$, as fórmulas matemáticas utilizadas por Noroozi et al. ${ }^{37}$ em 2001, ou mesmo as curvas Spline, formadas por tiras flexíveis ${ }^{3}$ e as

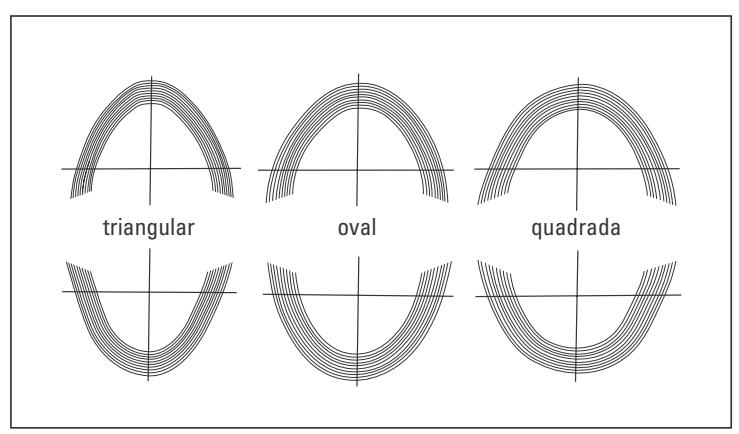

FIGURA 13 - Formas de Telles ${ }^{57}$.

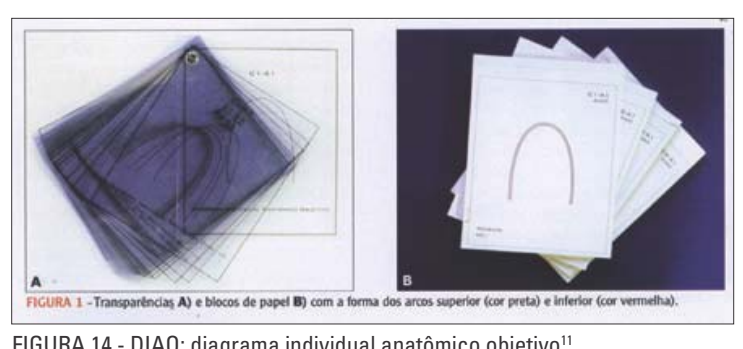

FIGURA 14 - DIA0: diagrama individual anatômico objetivo"1. 
séries de Fourier (VALENZUELA et al. ${ }^{61}$, 2002) foram empregadas em seus estudos, embora algumas delas fossem muito complexas.

Devido, então, à sua precisão e simplicidade matemática e por terem como representações gráficas segmento de curvas, as funções polinomiais foram aceitas por diversos autores ${ }^{17,21,32,38,44,58,59,63}$, que concordaram que os polinômios poderiam determinar, de maneira simples e simétrica, a forma do arco dentário.

$\mathrm{Na}$ literatura, encontraram-se autores ${ }^{17,32,44,63}$ que comprovaram a qualidade do polinômio de $4^{\circ}$ grau na determinação da forma dos arcos, enquanto Hechter ${ }^{21}$ preconizou o uso de um polinômio mais simples, o de $2^{\circ}$ grau (a função parabólica), para descrever as formas dos arcos dentários superiores e inferiores. Outros autores ${ }^{4,38}$ afirmaram que quanto maior o grau do polinômio mais precisa seria a determinação da curva representante da forma do arco dentário, com isso concordaram com a precisão do polinômio de $6^{\circ}$ grau na determinação da forma do arco dentário.
Baseando-se nesse conceito, Triviño e Vilella ${ }^{58}$ (2005), após a aplicação da função polinomial de $6^{\circ}$ grau em modelos de estudo, estabeleceram 6 formas distintas para a descrição da configuração da forma do arco dentário, ilustradas na figura 15 .

\section{DISCUSSÃO}

Com a revisão de literatura, observou-se que a forma do arco dentário vem sendo descrita pelos autores de várias maneiras diferentes e com distintas configurações. Frente a tantas controvérsias, pode-se notar que a preservação do equilíbrio entre estruturas musculares, ósseas e os dentes é a responsável pelo sucesso do tratamento ortodôntico, ou seja, pela estabilidade dos resultados. Assim, a forma inicial do arco dentário deve ser, necessariamente, mantida até o final do tratamento.

Em meio a vários estudos que abordaram o assunto forma do arco dentário, pôde-se observar que, no princípio, os autores tinham a preocupação de estabelecer ou encontrar uma forma que fosse considerada ideal para o arco dentário, com

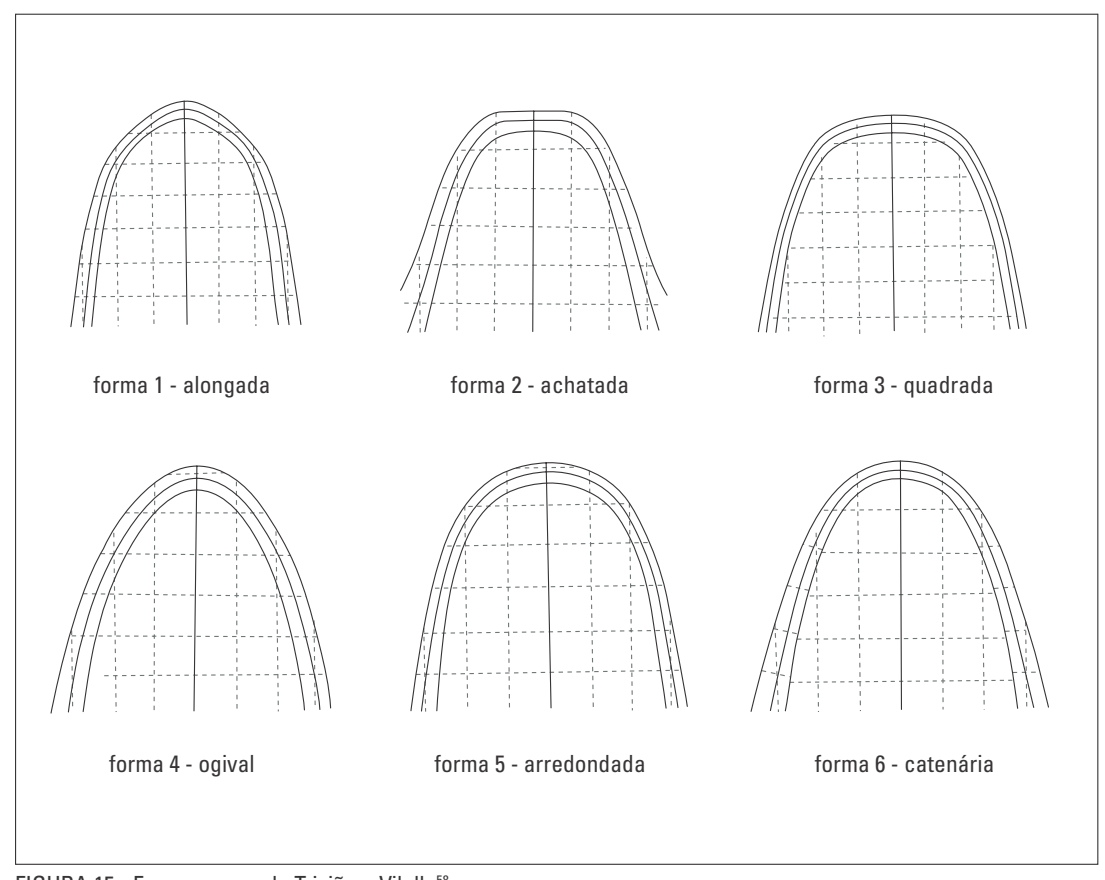

FIGURA 15 - Formas segundo Triviño e Vilella ${ }^{58}$. 
a qual a má oclusão seria corrigida, promovendo estética e função ao paciente. Com essa finalidade foram, inicialmente, desenvolvidos diagramas que forneciam somente uma configuração para o arco dentário, que apresentaria apenas uma variação quanto ao seu tamanho, sendo essa determinada pela soma dos 6 dentes ântero-inferiores ou pelas medidas craniofaciais.

Entretanto, o seguimento das pesquisas com objetivo de determinar a forma do arco dentário permitiu o emprego de métodos mais exatos, que compreendiam a associação da matemática aos programas de computadores e, como resultado, foram avaliadas duas ou mais formas representativas da configuração do arco dentário.

\section{CONCLUSÃO}

A manutenção da forma original do arco dentário - principalmente suas dimensões transversais - e, conseqüentemente, o equilíbrio entre as estruturas ósseas, musculares e tecidos moles são aspectos essenciais para atingir a estabilidade longínqua no tratamento ortodôntico.

Após o estudo da literatura, conclui-se que a maioria dos autores preconiza a utilização de formas de arcos individualizadas para cada paciente, sendo mais recomendáveis os diagramas que fornecem configurações distintas e não o emprego de uma forma de arco média, obtida a partir de medidas também médias.

Aconselha-se, então, a realização de estudos, nos quais serão avaliadas as possiveis formas representativas para o arco dentário com oclusão normal e a viabilidade da utilização de uma forma média no tratamento de uma má oclusão.

\title{
The lower dental arch form in the literature viewpoint
}

\begin{abstract}
Aim: The main purposes of this study were to analyze the literature related to a relevant clinic aspect, the dental arch form, emphasizing the importance of maintaining the original form in order to obtain stability after the orthodontic treatment, as well as to evaluate the methods used for establishing the dental arch form and the arch form diagrams proposed by many authors. Results and Conclusions: It was verified that there is some controversy regarding the indication of an ideal average form and the selection of an individualized form from diagrams which provide varied formats for the dental arch. However, some progress was observed in the methodologies applied in recent studies, which will possibly provide more accuracy in arch form determination in the near future.
\end{abstract}

Key words: Dental arch. Arch form. Stability. Orthodontics. 


\section{REFERÊNCIAS}

1. ANDREWS, L. F.; ANDREWS, W. A. Syllabus of the Andrews orthodontic philosophy. 9th ed. San Diego: Lawrence F. Andrews, 2001

2. ANGLE, E. H. Treatment of malocclusion of the teeth. 7th ed. Philadelphia: S. S. White Manufacting, 1907.

3. BeGOLE, E. A. Application of the cubic spline function in the description of dental arch form. J. Dent. Res., Chicago, v. 59 no. 9, p. 1549-1556, Sept. 1980

4. BIGGERSTAFF, R. H. Three variations in dental arch form estimated by a quadratic equation. J. Dent. Res., Chicago, v. 51 no. 5, p. 1509, Sept./Oct. 1972.

5. BOONE, G. N. Archwires designed for individual patients. Angle Orthod., Appleton, v. 33, no. 3, p. 178-185, July 1963.

6. BONWILL, W. G. A. The scientific articulation on the human teeth as founded on geometrical, mathematical and mechanical laws. Dent. Items Interest., Philadelphia, v. 21, no. 9, p. 617-643, Sept. 1885.

7. BRADER, A. C. Dental arch form related with intraoral forces: $\mathrm{PR}=$ C. Am. J. Orthod., St. Louis, v. 61, no. 6, p. 541-561, June 1972.

8. BRAUN, S.; HNAT, W. P.; FENDER, D. E.; LEGAN, H. L. The form of the human dental arch. Angle Orthod., Appleton, v. 68 , no. 1, p. 29-36, Feb.1998.

9. BURDI, A. R. Morphogenesis of mandibular dental arch shape in human embryos. J. Dent. Res., Chicago, v. 47, no. 1, p. 5058, Jan./Feb.1968.

10. CAMPIONI, A. P. F. Forma e dimensão dos arcos dentários. 2001. Trabalho de Conclusão de Curso (Especialização em Ortodontia e Ortopedia Facial dos Maxilares)-Associação Paulista de Cirurgiões-Dentistas, Presidente Prudente, 2001.

11. CAPELOZZA FILHO L.; CAPELOZZA, J. A. Z. DIAO: diagrama individual anatômico objetivo. Uma proposta para a escolha da forma dos arcos na técnica de Straight-Wire, baseada na individualidade anatômica e nos objetivos de tratamento. R. Clin. Ortodon. Dental Press, Maringá, v. 3, n. 5, p. 84-92, out./ nov. 2004

12. CASTELLANOS, E. C.; VIGORITO, J. W. Estudo das modificações nas dimensões transversais dos arcos dentários superior e inferior durante após o tratamento ortodôntico. Ortodontia, São Paulo, v. 10, n. 2, maio /ago. 1977.

13. CHUCK, G. C. Ideal arch form. Angle Orthod., Appleton, v. 4, no. 4, p. 312-327, Oct. 1934

14. CURRIER, J. H. A computerized geometric analysis of human dental arch form. Am. J. Orthod., St. Louis, v. 56, no. 2, p. 164-179, Aug. 1969.

15. CRUZ, A. D. L. et al. Long-term changes in arch form after orthodontic treatment and retention. Am. J. Orthod. Dentofacial Orthop., St. Louis, v. 107, no. 5, p. 518-529, May 1995.

16. ENGEL, G. A. Preformed arch wires: reability of fit. Am. J. Orthod., St Louis, v. 76, no. 5, p. 497-504, Nov. 1979.

17. FERRARIO, V. F. et al. Mathematical definition of the shape of dental arches in human permanent healthy dentitions. Eur. Orthod. Soc., Oxford, v.16, no. 4, p. 287-294, Aug 1994.

18. FIGÚN, M. E.; GARINO, R. R. Anatomia odontológica funcional e aplicada. 3. ed. Rio de Janeiro: Guanabara Koogan, 1994.

19. GUERRA, S. Dimensional changes of the dental arches: a review of the literature. Chron. Omaha Soc., Omaha, v. 35, no. 6, p. 154-156, Feb. 1972

20. HAWLEY, C. A. Determination of the normal arch and its application to Orthodontia. Dental Cosmos, Philadelphia, v. 47, no. 5, p. 541-552, May 1905

21. HECHTER, F. J. Symmetric and dental arch form of orthodontically treated patients. J. Canad. Dent. Assoc., Toronto, v. 44 no. 4, p. 173-184, Apr. 1978

22. HERREN, P.; OE, S. C. Die breitendifferenz zwischen den summierten oberen und unteren zahnkronen. Schweiz. Mschr. Zahnh. Bern, v. 81, no. 2, p. 113-125, Feb. 1971.
23. HOUSLEY, J. A. et al. Stability of transverse expansion in the mandibular arch. Am. J. Orthod. Dentofacial Orthop., St. Louis, v. 124, no. 3, p. 288-293, Sept. 2003.

24. HOWES, A. E. Arch width in the premolar region: still the major problem in Orthodontics. Am. J. Orthod., St Louis, v. 43, no. 1, p. 5-31, Jan. 1957.

25. HRDLICKA, A. The normal dental arch. Dental Cosmos, Philadelphia, v. 58, no. 9, p. 1029-1032, Sept. 1916.

26. INTERLANDI, S. New method for establishing arch form. J. Clin. Orthod., Boulder, v. 12, no. 12, p. 843-845, Dec. 1978.

27. IZARD, G. New method for the determination of the normal arch by the function of the face. Int. J. Orthod. Oral Surg. Radiog., St. Louis, v. 13, no. 7, p. 582-595, July 1927.

28. JOONDEPH, D. R.; RIEDEL, R. A. Retenção e recidiva. In: GRABER, M. T.; VANARSDALL, R. L. J. Ortodontia: princípios e técnicas atuais. 2. ed. Rio de Janeiro: Guanabara Koogan, 1996.

29. KANASHIRO, L. K.; VIGORITO, J. W. Estudo das formas e dimensões das arcadas dentárias superiores e inferiores em leucodermas, brasileiros, com maloclusão de Classe II - divisão $1^{\text {a }}$ e diferentes tipos faciais. Ortodontia, São Paulo, v. 33, n. 2, p. 8-18, maio/ ago. 2000

30. LEAR, C. S. C.; MOORREES, C. F. A. Buco-lingual muscle force and dental arch form. Am. J. Orthod., St. Louis, v. 56, no. 4, p. 379-393, Oct. 1969.

31. LITTLE, R. M. Stability and relapse of mandibular anterior aligment - first premolar extraction cases treated by tradicional Edgewise Orthodontics. Am. J. Orthod. Dentofacial Orthop., St. Louis, v. 80, no. 4, p. 349-365, Oct. 1981

32. LU, K. H. An orthogonal analysis of the form, simetry, and assymetry of the dental arch. Arch. Oral Biol., Oxford, v. 11, no. 11, p. 1057-1069, Nov. 1966

33. MAcCONAILL, M. A.; SCHER, E. A. The ideal form of the human dental arcade, with some prosthetic application. Dent. Rec., London, v. 69, no. 11, p. 285-302, Nov. 1949.

34. McLAUGHLIN, R.; BENNETT, J.; TREVISI, H. A forma do arco MBTTM e a seqüência do fio - parte 2. Rev. Dental Press Ortodon. Ortop. Facial, Maringá, v. 3, no. 4, p. 39-48, jul./ ago. 1998.

35. MONTI, A. E. Tratado de Ortodoncia. 3. ed. Buenos Aires: El Ateneo, 1958. v. 1, p. 221-236.

36. MUITINELLI, S.; MANFREDI, M.; COZZANI, M. A mathematicgeometric model to calculate variation in mandibular arch form. Eur. J. Orthod., Oxford, v. 22, no. 2, p. 113-125, Apr. 2000.

37. NOROOZI, H.; NIK, T. H.; SAEEDA, R. B. S. The dental arch form revisited. Angle Orthod., Appleton, v. 71, no. 5, p. 386389, Oct. 2001

38. PEPE, S. H. Polynomial and catenary curve fits to human dental arches. J. Dent. Res., Chicago, v. 54, no. 6, p. 1124-1132, Nov./Dec. 1975

39. PICOSSE, M. Contribuição ao estudo da morfologia do arco dentário superior nos brasileiros. 1955. Tese (Livre Docência em Anatomia)-Faculdade de Odontologia, Universidade de São Paulo, São Paulo, 1955

40. RABERIN, M. et al. Dimensions and form of dental arches in subjects with normal occlusions. Am. J. Orthod. Dentofacial Orthop., St. Louis, v. 104, no. 1, p. 67-72, July 1993.

41. RICKETTS, R. M. A detailed consideration of line of occlusion. Angle Orthod., Appleton, v. 48, no. 4, p. 274-282, Oct. 1978.

42. RICKETTS, R. M. Design of arch form and details for bracket placement. Denver: Rocky Mountain, 1979.

43. SALZMANN, J. A. An evaluation of retention and relapse following orthodontic therapy. Am. J. Orthod., St. Louis, v. 51, no. 10, p. $779-781$, Oct. 1965.

44. SANIN, C. et al. Arch length of the dental arch estimated by multiple regression. J. Dent. Res., Chicago, v. 49, no. 4 p. 885, July/Aug. 1970.

45. SAVOSTIN-ASLING, I. The geometric analysis of mandibular dental arch form. Ann. Dent., New York, v. 39, no. 1, p. 3-11, Spring 1980. 
46. SCOTT, J. H. The shape of the dental arches. J. Dent. Res. Chicago, v. 36, no. 6, p. 996-1003, Dec. 1957.

47. SHAPIRO, P. A. Mandibular dental arch form and dimension. Am. J. Orthod., St. Louis, v. 66, no. 1, p. 58-70, July 1974.

48. SPENGEMAN, W. G. Cuspid expansion. J. Pract. Orthod. New York, v. 11, no. 8, p. 414-417, Oct. 1968.

49. STATON, F. L. Arch predetermination and method of relating the predetermination to the malocclusion, to show the minimum tooth movement. Int. J. Orthod. Oral Surg. Radiog., St. Louis, v. 8, no. 12, p. 757-778, Dec. 1922.

50. STEADMAN, S. R. Changes of intermolar and intercuspid distances following orthodontic treatment. Angle Orthod., Appleton, v. 31, no. 4, p. 207-215, Oct. 1961

51. STEYN, C. L.; HARRIS, A. M. P. Anterior arch circunference adjustment - how much? Angle Orthod., Appleton, v. 66, no. 6, p. 457-462, June 1996.

52. STRANG, R. H. W. Factors of influence in producing a stable result in the treatment of malocclusion. Am. J. Orthod. Oral Surg., St. Louis, v. 32, no. 6, p. 313-332, June 1946.

53. STRANG, R. H. W. The fallacy of denture expansion as a treatment procedure. Angle Orthod., Appleton, v. 14, no. 1, p. 12-22, Jan. 1949.

54. STRANG, R. H. W. Factors associated with successful orthodontic treatment. Am. J. Orthod., St. Louis, v. 38, no. 10, p. 790-800, Oct. 1952.

55. STRANG, R. H. W. Highlights of sixty-four years in orthodontics. Angle Orthod., Appleton, v. 44, no. 2, p. 101-112, Apr. 1974.

56. SVED, A. Mathematics of the normal dental arch. Dental Cosmos, Philadelphia, v. 59, no. 11, p. 1116-1124, Nov. 1917.

57. TELLES, F. S. Diagramas de contorneamento. 1991. Trabalho de Conclusão de Curso (Especialização em Ortodontia)-Univer sidade Federal do Paraná, Curitiba, 1991.
58. TRIVIÑO T.: VILELLA, O. V. Formas e dimensões do arco dentário inferior. Rev. SBO, Rio de Janeiro, v. 5, n. 1, p. 19-28, set. 2005.

59. TSAI, H. Variations among the primary maxillary dental arch forms using a polynomial equation model. J. Clin. Pediatr. Dent., Birmingham, v. 27, no. 3, p. 267-270, Spring 2003.

60. VADEN, J. L.; DALE, J. G.; KLONTZ, H. A. Aparelho tipo Edgewise de Tweed-Merrifield: filosofia, diagnóstico e tratamento. In: GRABER, M. T.; VANARSDALL, R. L. J. Ortodontia: princípios e técnicas atuais. 2. ed. Rio de Janeiro: Guanabara Koogan, 1996.

61. VALENZUELA, P. A.; PARDO, M. A.; YEZIORO, S. Description of dental arch form using the fourier series. Int. J. Adult Orthodon. Orthognath. Surg., Chicago, v. 17, no. 1, p. 59-65, 2002.

62. VIGORITO, M. S. M. Estudo das formas e dimensões dos arcos dentários superiores em adolescentes brasileiros, leucodermas, portadores de oclusão normal. 1986. Dissertação (Mestrado em Ortodontia)-Universidade Metodista de São Paulo, São Bernardo do Campo, 1986.

63. WAKABAYASHI, K. et al. Development of the computerized dental cast form analyzing system - Three dimensional diagnosis of dental arch form and the investigation of measuring condition. Dent. Mater J., Tokyo, v. 16, no. 2, p. 180-190, Dec. 1997.

64. WALTER, D. C. Comparative changes in mandibular canine and first molar widths. Angle Orthod., Appleton, v. 32, no. 4 p. 232-240, Oct. 1962 .

65. WEINBERGER, B. W. Study of normal dental arches and normal occlusion. Dental Cosmos, Philadelphia, v. 56, no. 6 p. 665-680, June 1914

66. WHITE, L. W. Individualized ideal arches. J. Clin. Orthod., Boulder, v. 12, no. 11, p. 779-787, Nov. 1978.

67. WILLIAMS, P. N. Determining the shape of the normal arch. Dental Cosmos, Philadelphia, v. 59, no. 7, p. 695-708, July 1917.
Endereço para correspondência

Tarcila Triviño

Av. Nove de Julho, $n^{\circ} 5483,11^{\circ}$ andar, conjunto 111. Jd. Paulista CEP: 01.407-200 - São Paulo/SP

E-mail: tarcilatrivino@uol.com.br 